\title{
Cluster sets at ideal boundary points
}

\author{
By \\ Tatsuo Fuji'I'E \\ (Received May 15, 1971)
}

\section{Introduction}

The theory of cluster sets in plane regions has been studied in greater detail. To extend that theory to the case of Riemann surfaces it is natural to consider some kinds of compactifications of Riemann surfaces and define, in analogy of plane case, several kinds of cluster sets at ideal boundary points of the compactification. Then, one can expect that recent systematic studies of compactifications will give effective tools for the study of cluster sets at ideal boundary points.

In this paper we study cluster sets of an analytic mapping at Martin boundary points of a hyperbolic Riemann surface, where the cluster sets are defined with respect to Martin topology and to the fine topology. Especially in the case of a Fatou mapping, Wiener compactification, of which Martin compactification is a quotient space, is used to represent some of our cluster sets as sets of values of the mapping on certain subsets of its boundary ( $\$ 3)$.

For compactifications and related notions we refer to ConstantinescuCornea [4].

\section{§1. Definitions.}

We consider a non-constant analytic mapping $f$ of a Riemann surface $R$ into $R^{\prime} . \quad R$ is assumed hyperbolic in the sequel unless otherwise stated. Let $R^{*}$ be Martin compactification of $R$ and $R^{\prime *}$ a metrizable and resolutive compactification of $R^{\prime}$. We denote by $\Delta$ 
Martin boundary of $R$ and by $\Delta_{1}$ the set of minimal points in $\Delta$. To each point $p \in \Delta_{1}$ we associate a family (S) $_{p}$ of open sets in $R$ such that for each $G \in \mathbb{F}_{p}, R-G$ is thin at $p . \quad \mathbb{S}_{p}$ forms a filter base and the family $\{G \cup\{p\}\}$ constitutes the fine neighborhood system of $p$.

Fine cluster set at $p \in \Delta_{1}$ is defined as

$$
f^{\wedge}(p)=\bigcap_{G \in \mathfrak{S}_{p}} \overline{f(G)},
$$

where closure is taken in $R^{\prime *}$. When $f^{\wedge}(p)$ is one point we denote it by $f^{\prime}(p) . f^{\prime}(p)$ is a function of $p$ with values in $R^{\prime *}$ which is called a fine boundary function. Its domain of definition is denoted by $F(f)$.

For a point $p$ of $\Delta$ we define the following three cluster sets and range of values:

full cluster set

$$
C(f, p)=\bigcap_{r<0} \overline{f\left(U_{r} \cap R\right)}
$$

where $U_{r}$ denotes the $r$-neighborhood of $p$,

cluster set modulo $E$ of the fine boundary function $f^{\prime}$ of $f$ for a set $E$ on $\Delta$

$$
C_{E}^{*}(f, p)=\bigcap_{r>0} C_{E, r}^{*}(f, p)=\bigcap_{r \wedge 0} \overline{\bigcup_{q \in B} f_{r}^{\prime}(q)}
$$

where $B_{r}=F(f) \cap U_{r}-E-\{p\}$, and

essential cluster set of the fine boundary function of $f$

$$
C^{*}(f, p)=\bigcap_{E \in J} C_{E}^{*}(f, p)
$$

where $J$ is the collection of all sets on $\Delta$ of harmonic measure 0 . Closures in the first two cluster sets are taken in $R^{\prime *}$.

Range of values

$$
R(f, p)=\bigcap_{r>0} f\left(U_{r} \cap R\right)
$$


is the set of values which are assumed by $f$ in any neighborhood of $p$.

In the case that $R^{\prime}$ is Riemann sphere $S$ we have the following.

Theorem 1. Let $f$ be a meromorphic function on $R$ and $p$ be $a$ regular point of $\Delta$ with respect to Dirichlet problem. If $\Omega=C(f, p)$ $-C^{*}(f, p)$ is a non-empty open set, then each point of $\Omega$ is assumed infinitely often by $f$ in $R$ except for points belonging to a set of inner capacity 0.

Proof. It is sufficient to prove the theorem for each connected component $B$ of $\Omega$. Let $\alpha \in B$, then, by regularity of $p$, there exists a sequence $\left\{z_{n}\right\}$ tending to $p$ for which $f\left(z_{n}\right)$ tends to $\alpha$ and harmonic measure $\omega\left(z_{n}, \Delta \cap U_{r}, R\right)$ tends to 1 for all $r$-neighborhood $U_{r}$ ([4], [5]). Denote by $n_{f}(w)$ the valence function of $f$ and put $n_{f}=\sup _{w \in B} n_{f}(w)$, then the set $D_{n}=\left\{w \in B ; n_{f}(w) \leq n<n_{f}\right\}$ is a Borel set. And $D_{0}$ is of inner capacity $0([5])$.

Suppose now $\bigcup_{n<n_{f}} D_{n}$ is of positive inner capacity. Then, there exists an integer $n$ for which $D_{n-1}$ is of inner capacity 0 and $D_{n}$ is of inner capacity positive. Hence $D_{n}-D_{n-1}$ is of inner capacity positive and there exists a compact set $K$ of positive capacity in $D_{n}-D_{n-1}$. $D_{n}-D_{n-1}$ does not contain inner points, because its inner points can not be cluster values of $f$. Let $\alpha$ be a point of the kernel of $K$ which is not a branch point, that is, for any neighborhood $V=V(\alpha) V \cap K$ is of positive capacity. By taking $V$ sufficiently small, we may assume that $f^{-1}(V)$ consists of $n$ disks and non-compact regions. Then, $f^{-1}(V \cap K)$ is contained in the $n$ disks but not in the non-compact regions. We may further assume that $V$ is contained in $C(f, p)-C_{E, r}^{*}$ $(f, p)$ for sufficiently small $r$ and a set $E$ on $\Delta$ of harmonic measure 0 .

We take the non-compact component $C$ of $f^{-1}(V)$ which contains $z_{n}$, and estimate the harmonic measure

$$
\omega(w, V \cap K, V)=\omega(f(z), V \cap K, V) .
$$


Since

$$
\begin{aligned}
& \omega(f(z), V \cap K, V):\left\{\begin{array}{lll}
=0 & \text { on } & \partial C \\
\leq 1 & \text { in } & C
\end{array}\right. \\
& \omega(f(z), V \cap K, V) \leq I c 1 \text { in } C,
\end{aligned}
$$

where $I c$ denotes the inextremization to $C$. And

$$
\begin{aligned}
\omega\left(z, \Delta_{1}(C), R\right) & =E c I c 1 \geq I c 1 \\
& \geq \omega(f(z), V \cap K, V) \text { in } C,
\end{aligned}
$$

where $E c$ denotes the extremization $\left([3]\right.$ Satz $\left.15^{\prime}\right)$. Hence we have

$$
\omega(f(z), V \cap K, V) \leq \omega\left(z, \Delta_{1}(C), R\right) \quad \text { in } \quad C .
$$

Next, let $E_{0}=U_{r} \cap \Delta-F(f)$, then $E_{0}$ is of harmonic measure 0 because restriction of $f$ on each component of $U_{r} \cap R$ is a Fatou mapping (cf. Theorem 5). For a point $q \in U_{r} \cap \Delta_{1}-E_{0} \cup E$ ( $E$ and $r$ are chosen as above), there exists an open set $G \in \mathbb{S}_{q}$ such that $G \cap C=\phi$ because $f^{\wedge}(q)=f^{\prime}(q) \in C_{E, r}^{*}(f, p)$. Hence $q \notin \Delta_{1}(C)$ and $\Delta_{1}(C) \cap U_{r}$ is of harmonic measure 0 . Therefore,

(2) $\omega\left(z, \Delta_{1}(C), R\right)=\omega\left(z, \Delta_{1}(C)-U_{r}, R\right)$

$$
\leqq \omega\left(z, \Delta-U_{r}, R\right)=1-\omega\left(z, \Delta \cap U_{r}, R\right) .
$$

By (1) and (2) we have

$$
\omega(f(z), V \cap K, V) \leq 1-\omega\left(z, \Delta \cap U_{r}, R\right) \quad \text { in } \quad C .
$$

Especially,

$$
\omega\left(f\left(z_{n}\right), V \cap K, V\right) \leq 1-\omega\left(z_{n}, \Delta \cap U_{r}, R\right)
$$

for all $z_{n}$ of the sequence $\left\{z_{n}\right\}$. Let $z_{n}$ tend to $p$, then $1-\omega\left(z_{n}, \Delta \cap\right.$ $U_{r}, R$ ) tends to 0 , and we have

$$
\omega\left(f\left(z_{n}\right), V \cap K, V\right) \rightarrow 0 \text { for } z_{n} \rightarrow 0 .
$$


On the other hand, $f\left(z_{n}\right)$ tends to $\alpha$ when $z_{n}$ tends to $p$. So, we have

$$
\omega\left(f\left(z_{n}\right), V \cap K, V\right) \geq \delta>0
$$

for sufficiently large $n$.

(3) contradicts (4). Therefore, $D_{n}$ is of inner capacity 0 for all $n<n_{f}$. Each point of $C(f, p)-C^{*}(f, p)$ is assumed by $f$ by the same number of times ( $n_{f}$ times) except for points belonging to the set $\bigcup_{n<n_{f}} D_{n}$ of inner capacity 0 . But if $n_{f}$ is finite, $\bigcup_{n<n_{f}} D_{n}$ is relatively closed in the open set $\Omega=C(f, p)-C^{*}(f, p)$, and, to each point $w \in \Omega$ $-\bigcup_{n<n_{f}} D_{n}$, there exists a neighborhood $V$ contained in $\Omega-\bigcup_{n<n_{f}} D_{n}$, for which $f^{-1}(V)$ consists of $n_{f}$ disks. And so, $w$ can not be a cluster value of $f$ at $p$, which is contradiction. Consequently, $n_{f}$ is infinite and each point of $\Omega-\bigcup_{n<\infty} D_{n}$ is assumed by $f$ infinitely often. This completes the proof.

In the case that $R$ is parabolic, we compactify $R$ in the way of Alexandroff. Let $f$ be a meromorphic function in a neighborhood $G$ of $\infty$. We state the following theorem without proof, which is a generalization of Nakai's theorem (cf. [9]).

Theorem 2. $C(f, \infty)$ is of capacity 0 , or $f$ assumes every value on $f(G)$ infinitely often except for points belonging to a set of inner capacity 0.

To begin our study of cluster sets, we state the following important theorem of Plessner type which was proved by ConstantinescuCornea $([4])$ and Doob ([5]).

Theorem 3. (Constantinescu-Cornea and Doob). Let $f$ be an analytic mapping of $R$ into $R^{\prime}$ and $R^{\prime *}$ a metrizable and resolutive compactification of $R^{\prime}$. Then, for almost every (with respect to harmonic measure) point $p$ of $\Delta_{1}$, fine cluster set $f^{\wedge}(p)$ is one point or $R^{\prime *}$. 
By this theorem we classify points, $p$ of $\Delta_{1}$ as follows: $p \in \Delta_{1}$ is called

F-point if $f^{\wedge}(p)$ is one point, and we denote by $F(f)$ the set of $F$-points, and

I-point if $f^{\wedge}(p)$ is $R^{\prime *}$ (we say $f^{\wedge}(p)$ total), and we denote by $I(f)$ the set of $I$-points. A point $p \in \Delta$ is called

$H$-point if $R^{\prime}-R(f, p)$ is a polar set and denote by $H(f)$ the set of $H$-points. ${ }^{1)}$

The following theorems for an analytic mapping $f$ are also important for our study.

Theorem 4. (Constantinescu-Cornea $[4]) . \quad I(f)$ is of harmonic measure 0 if and only if $f$ is a Fatou mapping.

Theorem 5. (Constantinescu-Cornea [4]). For a hyperbolic $R$, if $f(R)$ is contained in a hyperbolic $R^{\prime} f$ is a Fatou mapping.

We study the cluster sets of $f$ separately according as $I(f)$ is of harmonic measure positive or zero.

\section{$\S 2$. The case that $I(f)$ is of harmonic measure positive.}

In this case $f$ is not a Fatou a mpping by Theorem 4. And we may assume $R^{\prime}$ parabolic because, otherwise $f$ will be a Fatou mapping and $I(f)$ will be of harmonic measure 0 by Theorem 4 and 5 . We consider Alexandroff compactification $R^{\prime *}$ which is metrizalbe and resolutive.

Lemma (Doob $[5])$. If $I(f)$ is of harmonic measure positive, then $R^{*}-f(R)$ is polar.

Proof. If $F_{0}=R^{\prime *}-f(R)$ is non polar, $f(R)$ is contained in the hyperbolic Riemann surface $R^{\prime}-F_{0}$. And $f$ is a Fatou mapping. This

1) F, I and $H$-points play the roles of Fatou, Plessner and Frostman points, respectively, at Martin boundary points. The former concepts, except $H$ points, do not reduce to the latter, however, in the case of the unit disk. 
contradicts the hypothesis.

Theorem 6. If $I(f)$ is of harmonic measure positive, then $I(f)$ $-H(f)$ is of harmonic measure 0 .

Proof. Let $p$ be a point of $I(f)$ outside a set of harmonic measure 0 . For every neighborhood $U$ of $p U \cap R$ contains a component $U_{i}$ such that the restriction of $f$ in $U_{i}$ is not a Fatou mapping [4]. Hence $R^{\prime *}-f\left(U_{i}\right)$ is polar by the lemma, so that $R^{\prime *}-f(U \cap R)$ is polar. For the $r$-neighborhood $U_{r}$ of $p$ we put $F_{r}=R^{\prime *}-f\left(U_{r} \cap R\right)$. Then $F_{r}$ is polar, and $R^{\prime}-R(f, p)=R^{\prime}-\bigcap_{n} f\left(U_{r_{n}} \cap R\right)=\bigcup_{n} F_{r_{n}}$ is polar. This shows that $p$ is an $H$-point.

If the set $f^{\prime}(F(f))$ of fine limits of $f$ is polar, we conclude directly by the above theorem and by the theorem of Lusin-Privalov type [4] that almost every point of $\Delta_{1}$ is an $H$-point. But the following theorem states sharper things.

Theorem 7. If the set $f^{\prime}(F(f))$ of fine limits of $f$ is polar, every regular point of $\Delta_{1}$ is an $H$-point.

Proof. Let $p$ be a point of $\Delta_{1}$ and $U$ be a neighborhood of $p$. Let $U \cap R=\bigcup_{i=1}^{\infty} U_{i}$ with connected components $U_{i}$, then $\bigcup_{i=1}^{\infty} \Delta_{1}\left(U_{i}\right)>$ $\Delta_{1} \cap U$. Since $p$ is regular by assumption $\Delta_{1} \cap U$ is of positive harmonic measure, and so, there exists a component $U_{i}$ such that $\Delta_{1}\left(U_{i}\right)$ is of harmonic measure positive.

Suppose $f^{\prime}(F(f))$ is polar, then, by the theorem of Lusin-Privalov type, $F(f)$ is of harmonic measure 0 . And, the full cluster set $C(f, p)$ must be total. Because, otherwise the restriction of $f$ on any component $U_{i}$ is a Fatou mapping and $\Delta_{1}\left(U_{i}\right) \subset F(f)$ except a set of harmonic measure 0 , which contradicts that there is at least one $\Delta_{1}\left(U_{i}\right)$ of positive harmonic measure.

Now, since $\overline{f(R)} \supset R^{\prime}$ and $F(f)$ is of harmonic measure $0, f$ is of type $B l$ in $R^{\prime}$ by Doob's theorem ([5] p. 541 cf. also [3]). Hence, 
$n_{f}(w)=n_{f}=\sup _{R^{\prime}} n_{f}(w)$ in $R^{\prime}$ except a set of capacity 0 . If $n_{f}$ is finite the set $E_{f}=\left\{w \in R^{\prime} ; n_{f}(w)<n_{f}\right\}$ is a closed set of capacity 0 . And, for a point $w \in R^{\prime}-E_{f}$, there exists a neighborhood $V=V(w)$ $C R^{\prime}-E_{f}$ such that $f^{-1}(V)$ consists of $n_{f}$ connected components which are relatively compact in $R$. This shows that $w$ is not a cluster value of $f$ at $p$ which contradicts that $C(f, p)$ is total. Therefore, $n_{f}$ is infinite and $E_{f}$ is polar, that is, $p$ is an $H$-point.

In the case that $R=(|z|<1)$, every point on $|z|=1$ is regular and minimal, and so, an $H$-point. And the above theorem corresponds to that of Collingwood and Lohwater $([1])$.

Remark. We pay our attention to the case that Martin boundary $\Delta$ of $R$ contains a singular point $p$, that is, a point of positive harmonic measure, and $R^{\prime}$ is parabolic. If $p$ is an $F$-point $f$ must be a constant by the theorem of Lusin-Privalov type, because $f^{\prime}(p)$ is a point of harmonic measure 0 of $R^{\prime *}$. If $p$ is an $I$-point $p$ is an $H$-point by Theorem 7. In the next section, we consider a Fatou mapping $f$ of $R$ into Riemann sphere, and we assume $\Delta$ does not contain a singular point unless $f$ is not a constant.

\section{§3. The case that $I(f)$ is of harmonic measure 0 .}

In this section we consider an analytic mapping $f$ of hyperbolic $R$ into Riemann sphere $S$. Since Theorem 3 and 4 are still valid in the case that $R^{\prime}=S, f$ is a Fatou mapping. Let $R_{W}^{*}$ be Wiener compactification, then $f$ is extended to a continuous mapping $f^{*}$ of $R_{W}^{*}$ into $S$. There exists a continuous mapping $\pi$ of $R_{W}^{*}$ into Martin compactification $R^{*}$ such that the restriction of $\pi$ on $R$ is identity ([6], [4]). For a point $p$ of $\Delta$, we put $W_{p}=\pi^{-1}(p), \Gamma_{p}=W_{p} \cap \Gamma$ and $\Lambda_{p}=W_{p}$ $-\Gamma_{p}=\Lambda \cap W_{p}$ for harmonic boundary $\Gamma$ and non-harmonic boundary $\Lambda$ of Wiener boundary $\Delta_{W}$. Then $\Gamma_{p}$ is a compact set. In the sequel we suppose $\Gamma_{p}$ is not empty. This is true, for instance, when $p$ is a regular point. Let $U_{n}$ be the $1 / n$-neighborhood of $p$ in $R^{*}$ and $V_{n}=$ $\pi^{-1}\left(U_{n}\right)$. Since $\pi^{-1}\left(\bar{U}_{n+1}\right)$ is a closed set contained in $\pi^{-1}\left(U_{n}\right)$ we have 


$$
\bigcap_{n} \pi^{-1}\left(\bar{U}_{n+1}\right)=\bigcap_{n} \pi^{-1}\left(U_{n}\right)
$$

where closure is taken in $R^{*}$. And,

$$
\bigcap_{n} \bar{V}_{n}=\bigcap_{n} V_{n}
$$

where closure is taken in $R_{W}^{*}$. We show

$$
W_{p}=\pi^{-1}(p)=\bigcap_{n} V_{n}
$$

Evidently $W_{p} \subset \bigcap_{n} V_{n}$. Suppose $q^{\prime} \in \bigcap_{n} V_{n}-W_{p}$ and denote $q=\pi\left(q^{\prime}\right)$. Let $N_{n}$ be a neighborhood of $q$ which does not contain $p$, then $M_{n}=$ $\pi^{-1}\left(N_{n}\right)$ is an open set containing $q^{\prime}$. So, $V_{m} \cap M_{n} \neq \phi$, for all $m$. But, since $q \neq p$ there exists a number $n$ such that $N_{n} \cap U_{m}=\phi$, which is a contradiction.

Theorem 8. For the full cluster set, we have

$$
C(f, p)=f^{*}\left(W_{p}\right)
$$

Proof. Since $\bar{V}_{n}=\overline{V_{n} \cap R}$ we have $f^{*}\left(\bar{V}_{n}\right)=f^{*}\left(\overline{V_{n} \cap R}\right)$. By continuity of $f^{*}$,

$$
f^{*}\left(\overline{V_{n} \cap R}\right)=\overline{f^{*}\left(V_{n} \cap R\right)}=\overline{f\left(V_{n} \cap R\right)} .
$$

Therefore we have

$$
f^{*}\left(\bar{V}_{n}\right)=\overline{f\left(V_{n} \cap R\right)}
$$

And, for the full cluster set, we have

$$
\begin{aligned}
C(f, p) & =\bigcap_{n} \overline{f\left(U_{n} \cap R\right)}=\bigcap_{n} \overline{f\left(V_{n} \cap R\right)}=\bigcap_{n} f^{*}\left(\bar{V}_{n}\right) \\
& =f^{*}\left(\bigcap_{n} \bar{V}_{n}\right) .
\end{aligned}
$$

We prove the last equality. Evidently, 


$$
\bigcap_{n} f^{*}\left(\bar{V}_{n}\right) \supset f^{*}\left(\bigcap_{n} \bar{V}_{n}\right)
$$

If $w \notin f^{*}\left(\bigcap_{n} \bar{V}_{n}\right)$

$$
f^{*-1}(w) \cap\left(\bigcap_{n} \bar{V}_{n}\right)=\bigcap_{n}\left(f^{*-1}(w) \cap \bar{V}_{n}\right)=\phi,
$$

and, since $\left\{f^{*-1}(w) \cap \bar{V}_{n}\right\}$ is a decreasing sequence of closed sets, there exists a number $n$ such that

$$
f^{*-1}(w) \cap \bar{V}_{n}=\phi,
$$

that is, $w \notin f^{*}\left(V_{n}\right)$. This shows

$$
\bigcap_{n} f^{*}\left(\bar{V}_{n}\right) \subset f^{*}\left(\bigcap_{n} \bar{V}_{n}\right)
$$

and completes the proof.

Now, we are going to characterize the essential cluster set of the fine boundary function of $f$ by $f^{*}\left(\Gamma_{p}\right)$. For a point $p \in \Delta$, we define the set

$$
L(U, \varepsilon, w)=\left\{p^{\prime} \in U \cap F(f) ; f^{\prime}\left(p^{\prime}\right) \in N_{\varepsilon}(w)\right\},
$$

where $U$ is a neighborhood of $p$ and $N_{\varepsilon}(w)$ is the $\varepsilon$-neighborhood of $w$ (cf. $[10]$ ).

\section{Lemma.}

$$
f^{*}\left(\Gamma_{p}\right)=\left\{\begin{array}{c}
w \in S ; L(U, \varepsilon, w) \text { is of positive harmonic } \\
\text { measure for all } \varepsilon>0 \text { and all } U
\end{array}\right\}
$$

Proof. Let $q$ be a point of $\Gamma_{p}$ and $w=f^{*}(q)$. By continuity of $f^{*}$ there exists, for an arbitrary neighborhood $N_{\varepsilon}$ of $w$, a neighborhood $\mathfrak{u}$ of $q$ such that $f^{*}(\mathfrak{H}) \subset N_{\varepsilon}$. And, since $q \in \mathfrak{U}$, there is a connected component $G$ of $R \cap \mathfrak{U}$ which is not of the class $S O_{H B}([7],[4])$. So, $\Delta_{1}(G)$ is of harmonic measure positive $([3])$, and the Fatou mapping $f$ has fine limits almost everywhere on $\Delta_{1}(G)$. Let $p^{\prime} \in \Delta_{1}(G) \cap F(f)$, then 


$$
f^{\prime}\left(p^{\prime}\right)=\bigcap_{V \in \Theta_{p},} \overline{f(V)} \subset \overline{f(G)} \subset \overline{f^{*}(\mathfrak{U})} \subset \bar{N}_{\varepsilon}
$$

This means that in an arbitrary neighborhood $N_{2 \varepsilon}$ of $w=f^{*}(q)$ there exists a fine limit $f^{\prime}\left(p^{\prime}\right)$ of $f$. And $p^{\prime}$ runs over the set $\Delta_{1}(G) \cap F(f)$ of harmonic measure positive which may be assumed to be contained in an arbitrarily given neighborhood $U$ of $p$ by taking $\mathfrak{U}$ small. Therefore, for $w=f^{*}(q) q \in \Gamma_{p}, L(U, \varepsilon, w)$ is of positive harmonic measure for all $\varepsilon>0$ and all $U$.

On the other hand, let $L(U, \varepsilon, w)$ be of harmonic measure positive for all $\varepsilon>0$ and all neighborhoods $U$ of $p$. Put $G=f^{-1}\left(N_{\varepsilon}\right)$ for the $\varepsilon$-neighborhood $N_{\varepsilon}$ of $w$. Then we have

$$
\Delta_{1}(G) \supset L(U, \varepsilon, w)
$$

because, for $p^{\prime} \in L(U, \varepsilon, w) f^{\prime}\left(p^{\prime}\right) \in N_{\varepsilon}$, so that $G=f^{-1}\left(N_{\varepsilon}\right) \in \mathcal{S}_{p^{\prime}}$ and $p^{\prime} \in \Delta_{1}(G)$. And, since $\Delta_{1}(U) \supset U \cap \Delta_{1} \supset L(U, \varepsilon, w)$ we have

$$
\Delta_{1}(G \cap U) \supset L(U, \varepsilon, w)
$$

and so, $\Delta_{1}(G \cap U)$ is of harmonic measure positive. This leads to existence of a component $D$ of $U \cap R$, for which $\Delta_{1}(D)$ is of harmonic measure positive. Hence $D$ is not of the class $S O_{H B}$, and we have

$$
(\bar{D}-(\overline{R-D})) \cap \Gamma \neq \phi
$$

where closures are taken in $R_{W}^{*}$. Here, we put $\Gamma_{U}=\Gamma \cap \overline{\pi^{-1}(U)}$, then it is a non-empty closed set containing $\bar{D} \cap \Gamma$. Since

$$
f^{*}(\Gamma \cap \bar{D}) \subset f^{*}(\bar{D}) \subset \overline{f(D)} \subset N_{2 \varepsilon}
$$

there exists a point $q$ in $\Gamma_{U}$ such that $f^{*}(q) \in N_{2 \varepsilon}$. Now, let $q_{n}$ be a point of $\Gamma_{U_{n}}$ such that $w_{n}=f^{*}\left(q_{n}\right) \in N_{\varepsilon_{n}}$. By making $\varepsilon_{n}$ tend to 0 and $\bar{V}_{n}=\overline{\pi^{-1}\left(U_{n}\right)}$ to $W_{p}$, we have a sequence $\left\{q_{n}\right\}$. Since $\Gamma_{U_{n}}$ is closed, $\overline{\left\{q_{n}\right\}} \subset \Gamma_{U_{n}}$ and $w \in f^{*}\left(\Gamma_{U_{n}}\right)$ for all $n$. Therefore, $w \in \bigcap_{n} f^{*}\left(\Gamma_{U_{n}}\right)=$ $f^{*}\left(\cap \Gamma_{U_{n}}\right)=f^{*}\left(\Gamma_{p}\right)$. 


\section{Theorem 9.}

$$
C^{*}(f, p)=f^{*}\left(\Gamma_{p}\right)
$$

Proof. First we recall that the boundary $\Delta$ does not contain a singular point in the present case, especially, $p$ is of harmonic measure 0 . Now, let

$$
w \in f^{*}\left(\Gamma_{p}\right),
$$

then $L(U, \varepsilon, w)$ is of harmonic measure positive for all $\varepsilon>0$ and all $U$, so that

$$
w \in \overline{p \in B} f^{\prime}(q)
$$

where $B=U \cap F(f)-E-\{p\}$ with an arbitrary set $E$ on $\Delta$ of harmonic measure 0. Hence,

$$
w \in \bigcap_{U}\left(\overline{\bigcup_{q \in B} f^{\prime}(q)}\right)=C_{E}^{*}(f, p) .
$$

Since $E$ is arbitrary,

$$
w \in \bigcap_{E \in J} C_{E}^{*}(f, p)=C^{*}(f, p) .
$$

On the contrary, if

$$
w \neq f^{*}\left(\Gamma_{p}\right)
$$

there exist $\varepsilon>0, U$ and $E \in J$ such that $N_{\varepsilon}(w) \cap\left\{\bigcup_{q \in B} f^{\prime}(q)\right\}=\phi$. Hence,

$$
w \notin \overline{\bigcup_{q \in B} f^{\prime}(q)}=C_{E}^{*}(f, p),
$$

and consequently,

$$
w \notin \bigcap_{E \in J} C_{E}^{*}(f, p)=C^{*}(f, p) .
$$

Thus we conclude that 


$$
f^{*}\left(\Gamma_{p}\right)=C^{*}(f, p)
$$

Theorem 8 and 9 suggest that properties of harmonic boundary will serve for the study of cluster sets. We shall prove a few theorems in the following as applications of the theorems.

Lemma. Let $p$ be a regular minimal point of $\Delta$, and $U$ be its neighborhood. Then, for $F=R-U$,

$$
1_{F}(z) \rightarrow 0 \quad \text { when } \quad z \rightarrow p
$$

where $1_{F}$ is the infimum of the class of superharmonic functions which are not smaller than 1 quasi-everywhere on $F$.

Proof. Let $f=\chi_{F \cap \Delta}$ be the characteristic function of $\bar{F} \cap \Delta$, where closure is taken in $R^{*}$. $f$ is resolutive and, by regularity of $p, H_{f}(z)$ $\rightarrow 0$ when $z \rightarrow 0$. Let $h_{1_{F}}=\inf _{s \in \mathbb{S}} s$, where $\mathfrak{S}$ is the class of superharmonic functions $s$ such that $s \geq 1_{F}$ outside a compact set $K s$. Then, since $h_{1_{F}} \leq H_{f}([4]), h_{1_{F}}(z) \rightarrow 0$ when $z \rightarrow 0$. But we have $0 \leq 1_{F} \leq h_{1_{F}}+\varepsilon P$ outside a compact set, with Green potential $P$ and $\varepsilon>0$ ([4]). And the potential $P$ tends to 0 at $p$, because the regular point $p$ belongs to the harmonic boundary of $R^{*}$. Therefore $1_{F}$ tends to 0 at $p$.

Lemma. Let $u$ be a continuous subharmonic function bounded above in a neighborhood of a regular minimal point $p$ of $\Delta$, then

$$
\max _{W_{p}} u=\max _{\Gamma_{p}} u
$$

Proof. Let $U_{n}$ be the $1 / n$-neighborhood of $p, V_{n}=\pi^{-1}\left(U_{n}\right)$ and $\Gamma_{n}=\Gamma \cap \bar{V}_{n}$. If $u$ satisfies the condition of the lemma in $U_{k}$, it is extended continuously on $\bar{V}_{n}$ for sufficiently large $n>k$. Because, for the component $C$ of $U_{k} \cap R$ belonging to $\mathbb{S}_{p}, p \in C^{*}=R^{*}-(\overline{R-C})$ and $u$ is extended continuously on $\pi^{-1}\left(C^{*}\right)$ (cf. [4] Satz 9.11). Let $u<M$ and put $m=\max _{\Gamma_{p}} u=\lim _{n \rightarrow \infty} \max _{\Gamma_{n}} u$, then there exists a number $n_{0}$ such 
that, for $n>n_{0}, \max _{r_{n}} u<m+\varepsilon<M$, for a given $\varepsilon>0$. By the preceding lemma, $1_{F_{n}} \rightarrow 0$ when $z \rightarrow p$, where $F_{n}=R-U_{n}$ for $n>n_{0}$. Put $\tilde{u}=$ $u-2 M 1_{F_{n}}$, then $\tilde{u}$ is a subharmonic function bounded above and

$$
\varlimsup \tilde{\lim } \leq\left\{\begin{array}{lll}
m+\varepsilon & \text { on } & \Gamma_{n} \\
0 & \text { on } & \partial V_{n} .
\end{array}\right.
$$

Hence,

$$
\tilde{u} \leq m+\varepsilon
$$

in $V_{n}\left(\left[4^{-}\right]\right)$. Since $1_{F_{n}} \rightarrow 0$ for $z \rightarrow p, 1_{F_{n}}<\varepsilon^{\prime}$ in $V_{m} \cap R$ for sufficiently small $V_{m}$. Therefore,

$$
u=\tilde{u}+2 M \varepsilon^{\prime} \leq m+\varepsilon+2 M \varepsilon^{\prime}
$$

in $V_{m} \cap R$. Since $u$ is continuous on $W_{p}$ and $\varepsilon$ and $\varepsilon^{\prime}$ are arbitrary, we have

$$
u \leq m \text { on } W_{p}
$$

and complete the proof.

Theorem 10. Let $p$ be a regular minimal point of $\Delta$, then $C(f, p)$ $-C^{*}(f, p)$ is an open set.

Proof. If $C(f, p)$ is total our assertion is evident. So, we assume $C(f, p)$ is not total. The proof is completed by showing that the boundary $\partial C(f, p)$ of $C(f, p)$ is contained in $C^{*}(f, p)$. We suppose that $w_{0} \in \partial C(f, p)-C^{*}(f, p)$. We may assume $w_{0}=0$ without loss of generality. Put $2 \rho$ the distance between $w_{0}$ and $C^{*}(f, p)$, and let $w_{1}$ be a point in the $\rho$-neighborhood of $w_{0}$ and outside $C(f, p)$. Then, $1 /\left|f(z)-w_{1}\right|$ is subharmonic and bounded above in $R \cap V_{n}$ for a certain $V_{n}$. And since $f\left(z_{n}\right)$ tends to $w_{0}=0$ for a sequence $\left\{z_{n}\right\}$ tending to $p$, $\max _{W_{p}}\left(1 /\left|f-w_{1}\right|\right) \geq 1 /\left|w_{1}\right|$. Therefore, by the preceding lemma, we have

$$
1 /\left|w_{1}\right| \leq \max _{W_{p}} 1 /\left|f-w_{1}\right| \leq \max _{\Gamma_{p}} 1 /\left|f-w_{1}\right|
$$




$$
\leq \max _{\Gamma_{p}} 1 / \mid\left(f|-| w_{1} \mid\right) \leq 1 /\left(2 \rho-\left|w_{1}\right|\right)
$$

Consequently, we have

$$
\left|w_{1}\right| \geq \rho
$$

which contradicts that $w_{1}$ belongs to the $\rho$-neighborhood of $w_{0}=0$.

Theorem 1 and 10 lead to the following

Theorem 11. Let $p$ be a regular minimal point of $\Delta$, then each point of $C(f, p)-C^{*}(f, p)$ is assumed by $f$ infinitely often in $R$ except for points belonging to a set of inner capacity 0 .

We notice that the values assumed by $f$ in any neighborhood of $p$ are those assumed by $f^{*}$ on $\Lambda_{p}$, that is, $R(f, p) \subset f^{*}\left(\Lambda_{p}\right)$.

\section{College of General Education, Kyoto University}

\section{References}

[1] Collingwood, E. F. and Lohwater, A. J. Applications of the theory of cluster sets to a class of meromorphic functions. Proc. Cambridge Phil. Soc. $\mathbf{5 3}$ (Part 1), (1957), 93-105.

bridge, (1966).

[3] Constantinescu, C. und Cornea, A.: Über den idealen Rand und einige seiner Anwendungen bei der Klassifikation der Riemannschen Flächen. Nagoya Math. J. 13, (1958), 169-233.

[4] — Ideale Ränder Riemannscher

Flächen. Berlin, (1963).

[5] Doob, J. L.: Conformally invariant cluster value theory. Illinois J. Math. 5, (1961), 521-547.

[6] Kusunoki, Y.: On a compactification of Green spaces. Dirichlet problem and theorems of Riesz type. J. Math. Kyoto Univ. 1, (1962), 385-402.

[7] Mori, S.: On a compactification of an open Riemann surface and its application. J. Math. Kyoto Univ. 1, (1961), 21-42.

[8] Noshiro, K.: Cluster sets, Berlin, (1960).

[9] Sario, L. and Noshiro, K.: Value distribution theory. Princeton, N. J., (1966).

[10] Weiss, M.L.: Cluster sets of bounded analytic functions from a Banach algebraic viewpoint. Ann. Acad. Sci. Fenn. 367, (1965). 